\title{
Controlled Lagrangians and Potential Shaping for Stabilization of Discrete Mechanical Systems
}

\author{
Anthony M. Bloch \\ Department of Mathematics \\ University of Michigan \\ Ann Arbor, MI 48109 \\ abloch@umich.edu \\ Jerrold E. Marsden \\ Control and Dynamical Systems \\ California Institute of Technology 107-81 \\ Pasadena, CA 91125 \\ marsdenecds.caltech.edu
}

\author{
Melvin Leok \\ Department of Mathematics \\ Purdue University \\ West Lafayette, IN 47907 \\ mleok@math.purdue.edu \\ Dmitry V. Zenkov \\ Department of Mathematics \\ North Carolina State University \\ Raleigh, NC 27695 \\ dvzenkoveunity.ncsu.edu
}

\begin{abstract}
The method of controlled Lagrangians for discrete mechanical systems is extended to include potential shaping in order to achieve complete state-space asymptotic stabilization. New terms in the controlled shape equation that are necessary for matching in the discrete context are introduced. The theory is illustrated with the problem of stabilization of the cartpendulum system on an incline. We also discuss digital and model predictive control.
\end{abstract}

\section{INTRODUCTION}

The method of controlled Lagrangians for stabilization of relative equilibria (steady state motions) originated in Bloch, Leonard, and Marsden [4] and was then developed in Auckly [1], Bloch, Leonard, and Marsden [5], [6], [7], Bloch, Chang, Leonard, and Marsden [8], and Hamberg [11], [12]. A similar approach for Hamiltonian controlled systems was introduced and further studied in the work of Blankenstein, Ortega, van der Schaft, Maschke, Spong, and their collaborators (see, e.g., [18] and related references). The two methods were shown to be equivalent in [9] and a nonholonomic version was developed in [20], [21], and [2].

According to the method of controlled Lagrangians, the original controlled system is represented as a new, uncontrolled Lagrangian system for a controlled Lagrangian, a modification of the original Lagrangian. The controlled Lagrangian is designed so that its associated energy has a maximum or minimum at the (relative) equilibrium to be stabilized. The time-invariant feedback control law is obtained by requiring that the new and old systems of equations of motion are equivalent. To obtain asymptotic stabilization, dissipation-emulating terms are added to the control input.

The method of controlled Lagrangians for discrete mechanical systems was introduced in Bloch, Leok, Marsden, and Zenkov [3]. In the present paper this formalism is further developed to include potential shaping which is used for complete state-space stabilization of equilibria. This study is motivated by the recent development of structure-preserving algorithms for numerical simulation of controlled systems. In particular, as the closed loop dynamics of a controlled Lagrangian system is itself Lagrangian, it is natural to adopt a variational discretization that exhibits good long-time numerical stability.

We carry out the matching procedure explicitly for discrete systems with two degrees of freedom and prove that we can asymptotically stabilize the equilibria of interest. The theoretical analysis is validated by simulating the discrete cart-pendulum system on an incline. When dissipation is added, the inverted pendulum configuration is asymptotically stabilized, as predicted. We then use the discrete controlled dynamics to construct a real-time model predictive controller with piecewise constant control inputs. This serves to illustrate how discrete mechanics can be naturally applied to yield digital controllers for mechanical systems.

The paper is organized as follows: In Sections II and III we review discrete mechanics and the method of controlled Lagrangians for stabilization of equilibria of mechanical systems. The discrete version of the potential shaping procedure and related stability analysis are discussed in Sections IV and V. The theory is illustrated with the discrete cartpendulum system. Simulations and the construction of the digital controller are presented in Sections VI and VII.

In a future publication we intend to treat discrete systems with nonabelian symmetries as well as systems with nonholonomic constraints.

\section{An Overview of Discrete Mechanics}

A discrete analogue of Lagrangian mechanics can be obtained by considering a discretization of the Hamilton principle; this approach underlies the construction of variational integrators. See Marsden and West [17], and references therein, for a more detailed discussion of discrete mechanics.

A key notion is that of the discrete Lagrangian, which is a map $L^{d}: Q \times Q \rightarrow \mathbb{R}$ that approximates the action integral along an exact solution of the Euler-Lagrange equations 
joining the configurations $q_{k}, q_{k+1} \in Q$,

$$
L^{d}\left(q_{k}, q_{k+1}\right) \approx \operatorname{ext}_{q \in \mathcal{C}([0, h], Q)} \int_{0}^{h} L(q, \dot{q}) d t,
$$

where $\mathcal{C}([0, h], Q)$ is the space of curves $q:[0, h] \rightarrow Q$ with $q(0)=q_{k}, q(h)=q_{k+1}$, and ext denotes extremum.

In the discrete setting, the action integral of Lagrangian mechanics is replaced by an action sum

$$
S^{d}\left(q_{0}, q_{1}, \ldots, q_{N}\right)=\sum_{k=0}^{N-1} L^{d}\left(q_{k}, q_{k+1}\right),
$$

where $q_{k} \in Q, k=0,1, \ldots, N$, is a finite sequence in the configuration space. The equations are obtained by the discrete Hamilton principle, which extremizes the discrete action given fixed endpoints $q_{0}$ and $q_{N}$. Taking the extremum over $q_{1}, \ldots, q_{N-1}$ gives the discrete Euler-Lagrange equations

$$
D_{1} L^{d}\left(q_{k}, q_{k+1}\right)+D_{2} L^{d}\left(q_{k-1}, q_{k}\right)=0
$$

for $k=1, \ldots, N-1$. This implicitly defines the update map $\Phi: Q \times Q \rightarrow Q \times Q$, where $\Phi\left(q_{k-1}, q_{k}\right)=\left(q_{k}, q_{k+1}\right)$ and $Q \times Q$ replaces the phase space $T Q$ of Lagrangian mechanics.

Since we are concerned with control, we need to consider the effect of external forces on Lagrangian systems. In the context of discrete mechanics, this is addressed by introducing the discrete Lagrange-d'Alembert principle (see Kane, Marsden, Ortiz, and West [14]), which states that

$$
\delta \sum_{k=0}^{n-1} L^{d}\left(q_{k}, q_{k+1}\right)+\sum_{k=0}^{n-1} F^{d}\left(q_{k}, q_{k+1}\right) \cdot\left(\delta q_{k}, \delta q_{k+1}\right)=0
$$

for all variations $\boldsymbol{\delta} \boldsymbol{q}$ of $\boldsymbol{q}$ that vanish at the endpoints. Here, $\boldsymbol{q}$ denotes the vector of positions $\left(q_{0}, q_{1}, \ldots, q_{N}\right)$, and $\boldsymbol{\delta} \boldsymbol{q}=\left(\delta q_{0}, \delta q_{1}, \ldots, \delta q_{N}\right)$, where $\delta q_{k} \in T_{q_{k}} Q$. The discrete one-form $F^{d}$ on $Q \times Q$ approximates the impulse integral between the points $q_{k}$ and $q_{k+1}$, just as the discrete Lagrangian $L^{d}$ approximates the action integral. We define the maps $F_{1}^{d}, F_{2}^{d}: Q \times Q \rightarrow T^{*} Q$ by the relations

$$
\begin{aligned}
& F_{2}^{d}\left(q_{0}, q_{1}\right) \delta q_{1}:=F^{d}\left(q_{0}, q_{1}\right) \cdot\left(0, \delta q_{1}\right), \\
& F_{1}^{d}\left(q_{0}, q_{1}\right) \delta q_{0}:=F^{d}\left(q_{0}, q_{1}\right) \cdot\left(\delta q_{0}, 0\right) .
\end{aligned}
$$

The discrete Lagrange-d'Alembert principle may then be rewritten as

$$
\begin{aligned}
& \delta \sum_{k=0}^{n-1} L^{d}\left(q_{k}, q_{k+1}\right) \\
& \quad+\sum_{k=0}^{n-1}\left[F_{1}^{d}\left(q_{k}, q_{k+1}\right) \delta q_{k}+F_{2}^{d}\left(q_{k}, q_{k+1}\right) \delta q_{k+1}\right]=0
\end{aligned}
$$

for all variations $\boldsymbol{\delta} \boldsymbol{q}$ of $\boldsymbol{q}$ that vanish at the endpoints. This is equivalent to the forced discrete Euler-Lagrange equations

$$
\begin{aligned}
D_{1} L^{d}\left(q_{k}, q_{k+1}\right) & +D_{2} L^{d}\left(q_{k-1}, q_{k}\right) \\
& +F_{1}^{d}\left(q_{k}, q_{k+1}\right)+F_{2}^{d}\left(q_{k-1}, q_{k}\right)=0 .
\end{aligned}
$$

\section{Matching AND Controlled LAGRAngians}

In the controlled Lagrangian approach, one considers a mechanical system with an uncontrolled (free) Lagrangian equal to kinetic energy minus potential energy. In the simplest setting, we modify the kinetic energy to produce a new controlled Lagrangian which describes the dynamics of the controlled closed-loop system. The method is extended by the incorporation of potential shaping in [8].

Suppose our system has configuration space $Q$ and a Lie group $G$ acts freely and properly on $Q$. It is useful to keep in mind the case in which $Q=S \times G$ with $G$ acting only on the second factor by the left group multiplication. For example, for the inverted planar pendulum on a cart, $Q=S^{1} \times \mathbb{R}$ with $G=\mathbb{R}$, the group of reals under addition (corresponding to translations of the cart).

Our goal is to control the variables lying in the shape space $Q / G$ using controls that act directly on the variables lying in $G .{ }^{1}$ For kinetic shaping, the controlled Lagrangian is constructed to be $G$-invariant, thus providing modified or controlled conservation laws. In this paper, we assume that $G$ is an abelian group.

The key modification of the Lagrangian involves changing the kinetic energy metric $g(\cdot, \cdot)$. The tangent space to $Q$ can be split into a sum of horizontal and vertical parts defined as follows: For each tangent vector $v_{q}$ to $Q$ at a point $q \in Q$, we can write a unique decomposition $v_{q}=\operatorname{Hor} v_{q}+\operatorname{Ver} v_{q}$, such that the vertical part is tangent to the orbits of the $G$-action and the horizontal part is metric-orthogonal to the vertical space, i.e., it is uniquely defined by the identity

$$
g\left(v_{q}, w_{q}\right)=g\left(\operatorname{Hor} v_{q}, \operatorname{Hor} w_{q}\right)+g\left(\operatorname{Ver} v_{q}, \operatorname{Ver} w_{q}\right)
$$

with $v_{q}$ and $w_{q}$ arbitrary tangent vectors to $Q$ at the point $q \in Q$. This choice of horizontal space coincides with that given by the mechanical connection; see, for example, Marsden [15].

For the kinetic energy of our controlled Lagrangian, we use a modified version of the right-hand side of equation (2). The potential energy remains unchanged. The modification consists of three ingredients:

1) a new choice of horizontal space, denoted $\mathrm{Hor}_{\tau}$,

2) a change $g \rightarrow g_{\sigma}$ of the metric on horizontal vectors,

3) a change $g \rightarrow g_{\rho}$ of the metric on vertical vectors.

Let $\xi_{Q}$ denote the infinitesimal generator corresponding to $\xi \in \mathfrak{g}$, where $\mathfrak{g}$ is the Lie algebra of $G$ (see Marsden [15] or Marsden and Ratiu [16]). Thus, for each $\xi \in \mathfrak{g}, \xi_{Q}$ is a vector field on the configuration manifold $Q$ and its value at a point $q \in Q$ is denoted $\xi_{Q}(q)$.

Definition 1: Let $\tau$ be a Lie-algebra-valued horizontal one-form on $Q$; that is, a one-form that annihilates vertical vectors. The $\tau$-horizontal space at $q \in Q$ consists of tangent vectors to $Q$ at $q$ of the form $\operatorname{Hor}_{\tau} v_{q}:=\operatorname{Hor} v_{q}-[\tau(v)]_{Q}(q)$, which also defines $v_{q} \mapsto \operatorname{Hor}_{\tau} v_{q}$, the $\tau$-horizontal projection. The $\tau$-vertical projection operator is defined by $\operatorname{Ver}_{\tau} v_{q}:=\operatorname{Ver} v_{q}+[\tau(v)]_{Q}(q)$.

\footnotetext{
${ }^{1}$ The shape space is $S$ in the case $Q=S \times G$.
} 
Definition 2: Given $g_{\sigma}, g_{\rho}$, and $\tau$, the associated controlled Lagrangian $L_{\tau, \sigma, \rho}$ is given by a modified kinetic minus the given potential energy, namely

$$
\begin{aligned}
L_{\tau, \sigma, \rho}\left(v_{q}\right)=\frac{1}{2}\left[g _ { \sigma } \left(\operatorname{Hor}_{\tau} v_{q},\right.\right. & \left.\operatorname{Hor}_{\tau} v_{q}\right) \\
& \left.+g_{\rho}\left(\operatorname{Ver}_{\tau} v_{q}, \operatorname{Ver}_{\tau} v_{q}\right)\right]-V(q) .
\end{aligned}
$$

The equations corresponding to this Lagrangian will be our closed-loop equations. The new terms appearing in those equations corresponding to the directly controlled variables are interpreted as control inputs. The modifications to the Lagrangian are chosen so that no new terms appear in the equations corresponding to the variables that are not directly controlled. We refer to this process as matching.

Once the form of the control law is derived using the controlled Lagrangian, the closed-loop stability of an equilibrium can be determined by energy methods, using any available freedom in the choice of $\tau, g_{\sigma}$ and $g_{\rho}$.

We can extend the method of controlled Lagrangians to the class of Lagrangian mechanical systems with potential energy that may break symmetry, i.e., we still have a symmetry group $G$ for the kinetic energy of the system but we now have a potential energy $V\left(x^{\alpha}, \theta^{a}\right)$ that need not be $G$-invariant (see [8]). Further, we consider a modification to the potential energy that also breaks symmetry in the group variables. Let the potential energy for the controlled Lagrangian be defined as

$$
V\left(x^{\alpha}, \theta^{a}\right)+V_{\varepsilon}\left(x^{\alpha}, \theta^{a}\right),
$$

where $V_{\varepsilon}$ is the modification-to be determined - that depends on a new real parameter $\varepsilon$.

For many systems it is sufficient to use the so-called simplified matching conditions [8]. For potential shaping in the setting where the simplified matching conditions hold we take $g_{\rho}=\rho g_{a b}$ where $\rho$ is a scalar constant. The controlled Lagrangian takes the form

$$
\begin{aligned}
& L_{\tau, \sigma, \rho, \varepsilon}(v)=L_{\tau, \sigma}(v)-V_{\varepsilon}\left(x^{\alpha}, \theta^{a}\right)+\frac{1}{2}(\rho-1) \times \\
& \quad g_{a b}\left(\dot{\theta}^{a}+\left(g^{a c} g_{\alpha c}+\tau_{\alpha}^{a}\right) \dot{x}^{\alpha}\right)\left(\dot{\theta}^{b}+\left(g^{b d} g_{\beta d}+\tau_{\beta}^{b}\right) \dot{x}^{\beta}\right),
\end{aligned}
$$

where

$$
L_{\tau, \sigma}(v)=L\left(x^{\alpha}, \dot{x}^{\beta}, \theta^{a}, \dot{\theta}^{a}+\tau_{\alpha}^{a} \dot{x}^{\alpha}\right)+\frac{1}{2} \sigma g_{a b} \tau_{\alpha}^{a} \tau_{\beta}^{b} \dot{x}^{\alpha} \dot{x}^{\beta} .
$$

This has sufficient generality to handle many examples of interest.

A basic example treated in earlier papers in the smooth setting is the pendulum on a cart. Let $s$ denote the position of the cart on the $s$-axis, $\phi$ denote the angle of the pendulum with the upright vertical, and $\psi$ denote the elevation angle of the incline, as in Figure 1. The configuration space for this system is $Q=S \times G=S^{1} \times \mathbb{R}$, with the first factor being the pendulum angle $\phi$ and the second factor being the cart position $s$. The velocity phase space, $T Q$, has coordinates $(\phi, s, \dot{\phi}, \dot{s})$. The length of the pendulum is $l$, the mass of the pendulum is $m$ and that of the cart is $M$.

The symmetry group $G$ of the kinetic energy of the pendulum-cart system is that of translation in the $s$ variable, so $G=\mathbb{R}$.

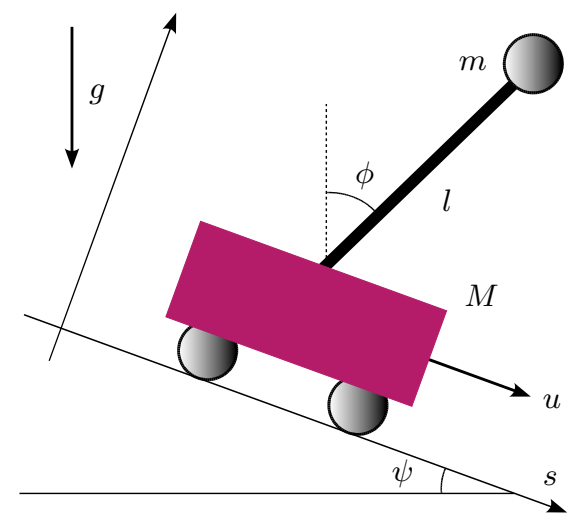

Fig. 1. The pendulum on a cart going down an inclined plane under gravity. The control force is in the direction $s$, the overall motion of the cart.

\section{Discrete Potential Shaping}

For simplicity, we consider systems with one shape and one group degree of freedom. We further assume that the configuration space $Q$ is the direct product of a onedimensional shape space $S$ and a one-dimensional Lie group $G$. The continuous-time Lagrangian $L: T Q \rightarrow \mathbb{R}$ and the form $\tau$ are

$$
L(\phi, s, \dot{\phi}, \dot{s})=\frac{1}{2}\left(\alpha \dot{\phi}^{2}+2 \beta(\phi) \dot{\phi} \dot{s}+\gamma \dot{s}^{2}\right)-V_{1}(\phi)-V_{2}(s)
$$

and

$$
\tau=k(\phi) \dot{\phi} \quad \text { with } \quad k(\phi)=-\frac{\beta(\phi)}{\sigma \gamma} .
$$

This Lagrangian (3) satisfies the simplified matching conditions of [8].

The continuous-time controlled Lagrangian $L_{\tau, \sigma, \rho, \varepsilon}$ : $T Q \rightarrow \mathbb{R}$ becomes

$$
\begin{aligned}
& L_{\tau, \sigma, \rho, \varepsilon}(\phi, s, \dot{\phi}, \dot{s})=L(\phi, s, \dot{\phi}, \dot{s}+k(\phi) \dot{\phi})+\frac{1}{2} \sigma \gamma(k(\phi) \dot{\phi})^{2} \\
& \quad+\frac{1}{2}(\rho-1) \gamma(\dot{s}+(\sigma-1) k(\phi) \dot{\phi})^{2}+V_{2}(s)-V_{\varepsilon}(y), \quad \text { (4) }
\end{aligned}
$$

where

$$
y=s-\int_{\phi_{e}}^{\phi} \frac{1}{\gamma}\left(\frac{1}{\sigma}-\frac{\rho-1}{\rho}\right) \beta(z) d z,
$$

the function $V_{\varepsilon}(y)$ is arbitrary, and $\left(\phi_{e}, s_{e}\right)$ is the equilibrium of interest. As in Bloch, Chang, Leonard, and Marsden [8], the kinetic energies in (3) and (4) are $G$-invariant.

For the cart-pendulum system, $\alpha, \beta(\phi), \gamma, V_{1}(\phi)$, and $V_{2}(s)$ are $\alpha=m l^{2}, \beta(\phi)=m l \cos (\phi-\psi), \gamma=M+m$, $V_{1}(\phi)=-m g l \cos \phi$, and $V_{2}(s)=-\gamma g s \sin \psi$. Note that $\alpha \gamma-\beta^{2}(\phi)>0$.

In discretizing the method of controlled Lagrangians, we combine formulae (1), (3), and (4). In the rest of this paper, we will adopt the notations

$$
q_{k+1 / 2}=\frac{q_{k}+q_{k+1}}{2}, \quad \Delta q_{k}=q_{k+1}-q_{k} .
$$

This allows us to construct a second-order accurate discrete Lagrangian and discrete controlled Lagrangian as

$$
\begin{aligned}
L^{d}\left(q_{k}, q_{k+1}\right) & =h L\left(q_{k+1 / 2}, \Delta q_{k} / h\right), \\
L_{\tau, \sigma, \rho, \varepsilon}^{d}\left(q_{k}, q_{k+1}\right) & =h L_{\tau, \sigma, \rho, \varepsilon}\left(q_{k+1 / 2}, \Delta q_{k} / h\right),
\end{aligned}
$$


with $q_{k}=\left(\phi_{k}, s_{k}\right)$.

The discrete dynamics is governed by the equations

$$
\begin{aligned}
& \frac{\partial L^{d}\left(q_{k}, q_{k+1}\right)}{\partial \phi_{k}}+\frac{\partial L^{d}\left(q_{k-1}, q_{k}\right)}{\partial \phi_{k}}=0, \\
& \frac{\partial L^{d}\left(q_{k}, q_{k+1}\right)}{\partial s_{k}}+\frac{\partial L^{d}\left(q_{k-1}, q_{k}\right)}{\partial s_{k}}=u_{k},
\end{aligned}
$$

where $u_{k}$ is the control input.

The dynamics associated with (5) is amended by the term $w_{k}$ in the discrete shape equation:

$$
\begin{aligned}
& \frac{\partial L_{\tau, \sigma, \rho, \varepsilon}^{d}\left(q_{k}, q_{k+1}\right)}{\partial \phi_{k}}+\frac{\partial L_{\tau, \sigma, \rho, \varepsilon}^{d}\left(q_{k-1}, q_{k}\right)}{\partial \phi_{k}}=w_{k}, \\
& \frac{\partial L_{\tau, \sigma, \rho, \varepsilon}^{d}\left(q_{k}, q_{k+1}\right)}{\partial s_{k}}+\frac{\partial L_{\tau, \sigma, \rho, \varepsilon}^{d}\left(q_{k-1}, q_{k}\right)}{\partial s_{k}}=0 .
\end{aligned}
$$

This term $w_{k}$ is important for matching systems (6), (7) and (8), (9). The presence of the terms $w_{k}$ represents an interesting (but manageable) departure from the continuous theory. Let

$$
J_{k}=\rho \gamma\left(\Delta s_{k} / h-(\sigma-1) k\left(\phi_{k+\frac{1}{2}}\right) \Delta \phi_{k} / h\right) .
$$

The following statement is proved by a straightforward calculation:

Theorem 3: The dynamics (6), (7) is equivalent to the dynamics (8), (9) if and only if $u_{k}$ and $w_{k}$ are given by

$$
\begin{aligned}
u_{k}= & -\frac{h}{2}\left[V_{2}^{\prime}\left(s_{k+\frac{1}{2}}\right)+V_{2}^{\prime}\left(s_{k-\frac{1}{2}}\right)\right] \\
& +\frac{h}{2 \rho}\left[V_{\varepsilon}^{\prime}\left(s_{k+\frac{1}{2}}\right)+V_{\varepsilon}^{\prime}\left(s_{k-\frac{1}{2}}\right)\right] \\
& +\frac{\gamma \Delta \phi_{k} k\left(\phi_{k+1 / 2}\right)-\gamma \Delta \phi_{k-1} k\left(\phi_{k-1 / 2}\right)}{h},
\end{aligned}
$$

and

$$
\begin{aligned}
w_{k}= & -\left(1-\sigma+\frac{\sigma}{\rho}\right)\left(k\left(\phi_{k+\frac{1}{2}}\right)\left[-\gamma \rho J_{k}+\frac{h}{2} V_{\varepsilon}^{\prime}\left(y_{k+\frac{1}{2}}\right)\right]\right. \\
& +k\left(\phi_{k-\frac{1}{2}}\right)\left[\gamma \rho J_{k-1}+\frac{h}{2} V_{\varepsilon}^{\prime}\left(y_{k-\frac{1}{2}}\right)\right] \\
& \left.-k^{\prime}\left(\phi_{k+\frac{1}{2}}\right) J_{k} \Delta \phi_{k}-k^{\prime}\left(\phi_{k-\frac{1}{2}}\right) J_{k-1} \Delta \phi_{k-1}\right) .
\end{aligned}
$$

Remark. The terms $w_{k}$ vanish when $\beta(\phi)=$ const as they become proportional to the left-hand side of equation (9).

\section{Stabilization of the Discrete Controlled SYSTEM}

The stability analysis in this paper is done by means of an analysis of the spectrum of the linearized discrete equations. We assume that the equilibrium to be stabilized is $\left(\phi_{k}, s_{k}\right)=$ $(0,0)$.

Theorem 4: The equilibrium $\left(\phi_{k}, s_{k}\right)=(0,0)$ of equations (8) and (9) is spectrally stable if

$$
\begin{gathered}
-\frac{\beta^{2}(0)}{\alpha \gamma-\beta^{2}(0)}<\sigma<0, \quad \rho<0, \quad \text { and } \quad V_{\varepsilon}^{\prime \prime}(0)<0 \\
\text { Proof: The linearized discrete equations are } \\
\frac{\partial \mathcal{L}_{\tau, \sigma, \rho, \varepsilon}^{d}\left(q_{k}, q_{k+1}\right)}{\partial \phi_{k}}+\frac{\partial L_{\tau, \sigma, \rho, \varepsilon}^{d}\left(q_{k-1}, q_{k}\right)}{\partial \phi_{k}}=0 \\
\frac{\partial \mathcal{L}_{\tau, \sigma, \rho, \varepsilon}^{d}\left(q_{k}, q_{k+1}\right)}{\partial s_{k}}+\frac{\partial \mathcal{L}_{\tau, \sigma, \rho, \varepsilon}^{d}\left(q_{k-1}, q_{k}\right)}{\partial s_{k}}=0
\end{gathered}
$$

where $\mathcal{L}_{\tau, \sigma, \rho, \varepsilon}^{d}\left(q_{k}, q_{k+1}\right)$ is the quadratic approximation of $L_{\tau, \sigma, \rho, \varepsilon}^{d}$ at the equilibrium (i.e., $\beta(\phi), V_{1}(\phi)$, and $V_{\varepsilon}(y)$ in $L_{\tau, \sigma, \rho, \varepsilon}^{d}$ are replaced by $\beta(0), \frac{1}{2} V_{1}^{\prime \prime}(0) \phi^{2}$, and $\frac{1}{2} V_{\varepsilon}^{\prime \prime}(0) y^{2}$, respectively). Note the absence of the term $w_{k}$ in equation (12).

The linearized dynamics preserves the quadratic approximation of the discrete energy

$$
\begin{gathered}
\frac{\alpha \gamma \sigma^{2}-\beta(0)^{2}(\sigma-1)(\rho(\sigma-1)-\sigma)}{2 \gamma \sigma^{2} h} \Delta \phi_{k}^{2} \\
+\frac{\beta(0) \rho(\sigma-1)}{\sigma h} \Delta \phi_{k} \Delta s_{k}+\frac{\gamma \rho}{2 h} \Delta s_{k}^{2} \\
\quad+\frac{h}{2} V_{1}^{\prime \prime}(0) \phi_{k+\frac{1}{2}}^{2}+\frac{h}{2} V_{\varepsilon}^{\prime \prime}(0) x_{k+\frac{1}{2}}^{2},
\end{gathered}
$$

where

$$
x=s+\left(\frac{\rho-1}{\rho}-\frac{1}{\sigma}\right) \frac{\beta(0)}{\gamma} \phi .
$$

Since $V_{1}^{\prime \prime}(0)$ is negative, the equilibrium $\left(\phi_{k}, s_{k}\right)=(0,0)$ of equations (12) and (13) is stable if the function (14) is negative-definite. The latter requirement is equivalent to conditions (11). The spectrum of the linearized discrete dynamics in this case belongs to the unit circle.

Remarks. Spectral stability in this situation is not sufficient to conclude nonlinear stability. The stability conditions (11) are identical to the stability conditions of the corresponding continuous-time system.

Following [8], we now modify the control input (10) by adding the discrete dissipation-emulating term

$$
-\frac{D\left(\Delta y_{k-1}+\Delta y_{k}\right)}{h}
$$

in order to achieve the asymptotic stabilization of the equilibrium $\left(\phi_{k}, s_{k}\right)=(0,0)$. In the above, $D$ is a constant. The linearized discrete dynamics becomes

$$
\begin{array}{r}
\frac{\partial \mathcal{L}_{\tau, \sigma, \rho, \varepsilon}^{d}\left(q_{k}, q_{k+1}\right)}{\partial \phi_{k}}+\frac{\partial L_{\tau, \sigma, \rho, \varepsilon}^{d}\left(q_{k-1}, q_{k}\right)}{\partial \phi_{k}} \\
=-\left(\frac{\rho-1}{\rho}-\frac{1}{\sigma}\right) \frac{\beta(0)}{\gamma} \frac{D\left(\Delta x_{k-1}+\Delta x_{k}\right)}{h}, \\
\frac{\partial \mathcal{L}_{\tau, \sigma, \rho, \varepsilon}^{d}\left(q_{k}, q_{k+1}\right)}{\partial s_{k}}+\frac{\partial \mathcal{L}_{\tau, \sigma, \rho, \varepsilon}^{d}\left(q_{k-1}, q_{k}\right)}{\partial s_{k}} \\
=-\frac{D\left(\Delta x_{k-1}+\Delta x_{k}\right)}{h} .
\end{array}
$$

Theorem 5: The equilibrium $\left(\phi_{k}, s_{k}\right)=(0,0)$ of equations (16) and (17) is asymptotically stable if conditions (11) are satisfied and $D$ is positive.

Proof: Multiplying equations (16) and (17) by $\left(\Delta \phi_{k-1}+\Delta \phi_{k}\right) / 2$ and $\left(\Delta s_{k-1}+\Delta s_{k}\right) / 2$, respectively, we obtain

$$
E_{k, k+1}=E_{k-1, k}+\frac{D h}{4}\left(\frac{\Delta x_{k-1}}{h}+\frac{\Delta x_{k}}{h}\right)^{2},
$$

where $E_{k, k+1}$ is the quadratic approximation of the discrete energy (14). Recall that $E_{k, k+1}$ is negative-definite. It is possible to show that, in some neighborhood of $\left(\phi_{k}, s_{k}\right)=$ $(0,0)$, the quantity $\Delta x_{k-1}+\Delta x_{k} \not \equiv 0$ along a solution of 
equations (16) and (17) unless this solution is the equilibrium $\left(\phi_{k}, s_{k}\right)=(0,0)$. Therefore, $E_{k, k+1}$ increases along nonequilibrium solutions of (16) and (17). Since equations (16) and (17) are linear, this is only possible if the spectrum of (16) and (17) is inside the open unit disk, which implies asymptotic stability of the equilibrium of both linear system (16) and (17) and nonlinear system (6) and (7) with discrete dissipation-emulating term (15) added to $u_{k}$.

\section{Simulations}

Simulating the discrete behavior of the controlled Lagrangian system involves viewing equations (6) and (9) as an implict update map $\Phi:\left(q_{k-2}, q_{k-1}\right) \mapsto\left(q_{k-1}, q_{k}\right)$. This presupposes that the initial conditions are given in the form $\left(q_{0}, q_{1}\right)$; however it is generally preferable to specify the initial conditions as $\left(q_{0}, \dot{q}_{0}\right)$. This is achieved by solving the boundary condition

$$
\frac{\partial L}{\partial \dot{q}}\left(q_{0}, \dot{q}_{0}\right)+D_{1} L^{d}\left(q_{0}, q_{1}\right)+F_{1}^{d}\left(q_{0}, q_{1}\right)=0
$$

for $q_{1}$. Once the initial conditions are expressed in the form $\left(q_{0}, q_{1}\right)$, the discrete evolution can be obtained using the implicit update map $\Phi$.

In Figure 2, we present a MATLAB simulation of discrete controlled dynamics of the cart-pendulum system in the absence of dissipation.
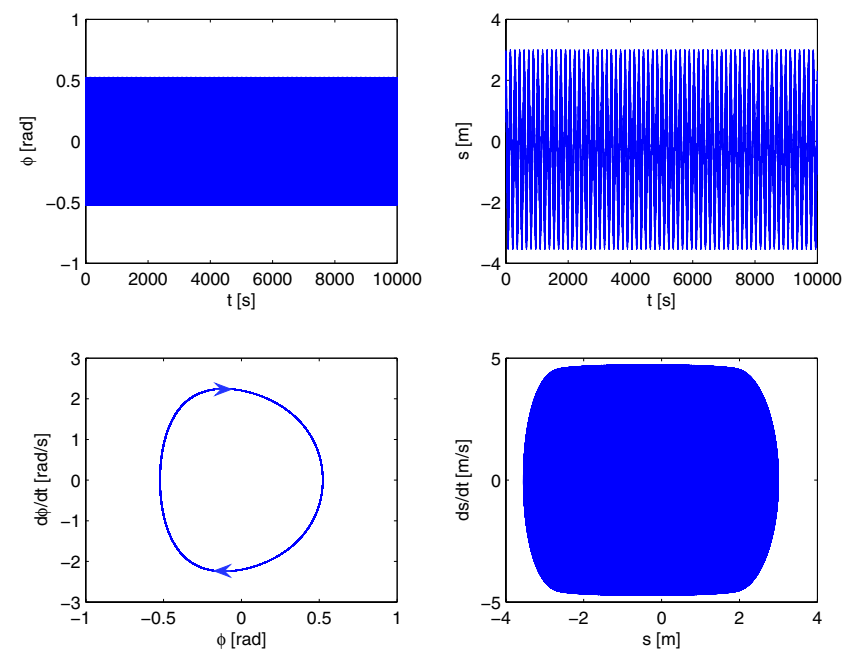

Fig. 2. Discrete controlled dynamics without dissipation. The discrete controlled system stabilizes the motion about the equilibrium; since there is no dissipation, the oscillations are sustained.

Here, $h=0.05 \mathrm{sec}, m=0.14 \mathrm{~kg}, M=0.44 \mathrm{~kg}, l=$ $0.215 \mathrm{~m}$, and $\psi=\frac{\pi}{9}$ radians. Our goal is to regulate the cart at $s=0$ and the pendulum at $\phi=0$. The control gains are chosen to be $\kappa=20, \rho=-0.02$, and $\varepsilon=0.00001$. It is worth noting that the discrete dynamics remain bounded near the desired equilibrium, and this behavior persists even for significantly longer simulation runs involving $10^{6}$ timesteps. The exceptional stability of the discrete controlled trajectory can presumably be understood in terms of the bounded energy oscillations characteristic of symplectic and variational integrators.

When dissipation is added, we obtain an asymptotically stabilizing control law, as illustrated in Figure 3. This is consistent with the stability analysis of Section V.
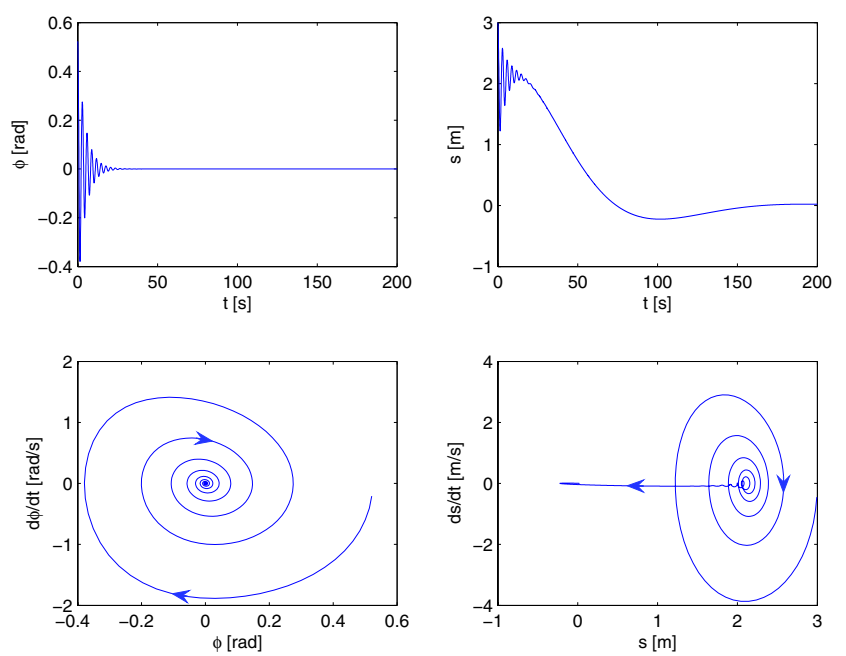

Fig. 3. Discrete controlled dynamics with dissipation. Here the oscillations die out and the cart converges to the desired point $s=0$.

\section{Model Predictive Controller}

We now explore the use of the forced discrete EulerLagrange equations as the model in a real-time model predictive controller, with piecewise constant control forces. Algorithm 1 below describes the details of the procedure.

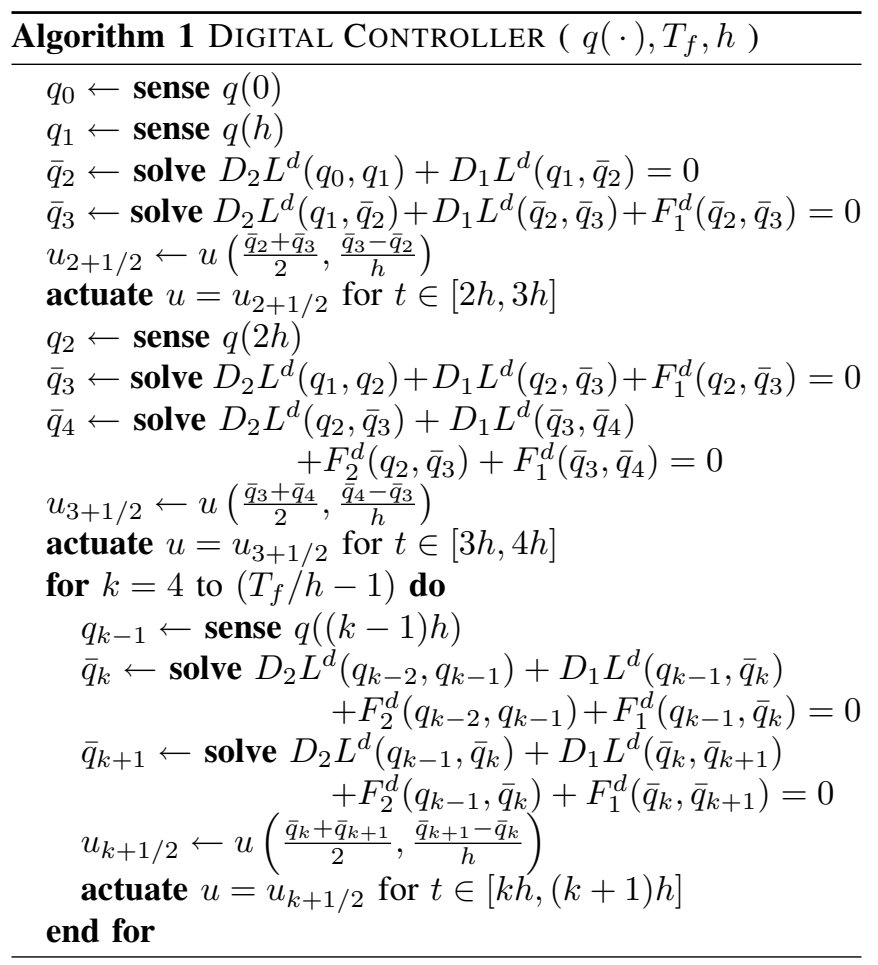


The digital controller uses the position information it senses for $t=-2 h,-h$ to estimate the positions at $t=0, h$ during the time interval $t=[-h, 0]$. This allows it to compute a symmetric finite difference approximation to the continuous control force $u(\phi, s, \dot{\phi}, \dot{s})$ at $t=h / 2$ using the approximation

$$
u_{1 / 2}=u\left(\frac{\bar{\phi}_{0}+\bar{\phi}_{1}}{2}, \frac{\bar{s}_{0}+\bar{s}_{1}}{2}, \frac{\bar{\phi}_{1}-\bar{\phi}_{0}}{h}, \frac{\bar{s}_{1}-\bar{s}_{0}}{h}\right),
$$

where the overbar indicates that the position variable is being estimated by the numerical model. This control is then applied as a constant control input for the time interval $[0, h]$. This algorithm can be implemented in real-time if the two forward solves can be computed within the time interval $h$.

The initialization of the discrete controller is somewhat involved, since the system is unforced during the time interval $[0,2 h]$ while the controller senses the initial states, and computes the appropriate control forces.

The numerical simulation of the digital controller is shown in Figure 4. We see that the system is asymptotically stabilized in both the $\phi$ and $s$ variables.
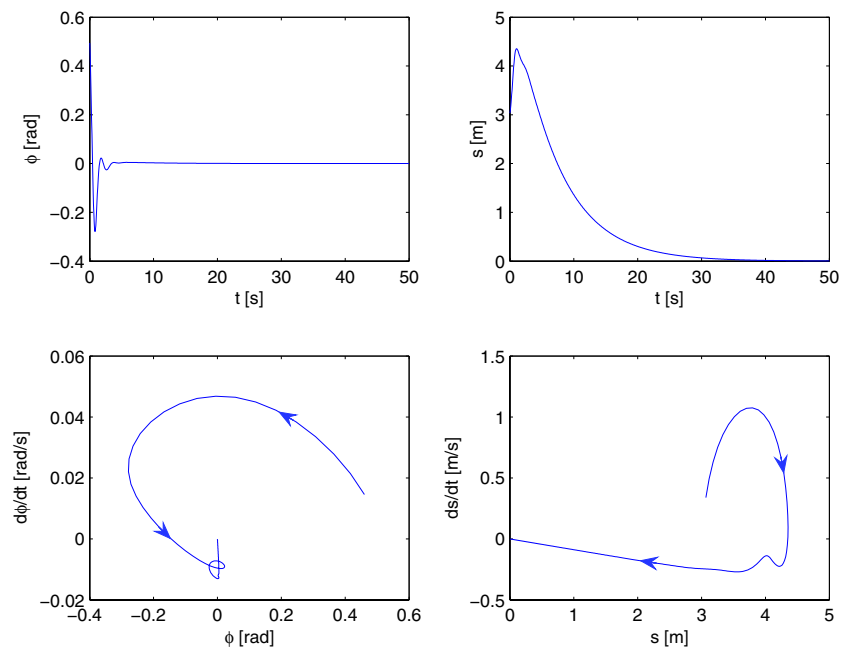

Fig. 4. The discrete real-time piecewise constant model predictive controller stabilizes $\phi$ and $s$ to zero.

\section{CONCLUSIONS}

In this paper we have introduced potential shaping techniques for discrete systems and have shown that these lead to an effective numerical implementation for stabilization in the case of the discrete cart-pendulum model. The method in this paper is related to other discrete methods in control that have a long history; recent papers that use discrete mechanics in the context of optimal control and celestial navigation are [10], [13], and [19]. The full theory of discrete controlled Lagrangians will be developed in a forthcoming paper.

\section{ACKNOWLEDGMents}

The authors would like to thank the reviewers for helpful remarks. The research of AMB was supported by NSF grants DMS-0305837, DMS-0604307, and CMS-0408542. The research of ML was partially supported by NSF grant DMS-0504747 and a University of Michigan Rackham faculty grant. The research of JEM was partially supported by AFOSR Contract FA9550-05-1-0343. The research of DVZ was partially supported by NSF grants DMS-0306017 and DMS-0604108.

\section{REFERENCES}

[1] Auckly, D., L. Kapitanski, \& W. White, Control of Nonlinear Underactuated Systems, Commun. Pure Appl. Math. 53, 2000, 354-369.

[2] Bloch, A. M., Nonholonomic Mechanics and Control, Interdisciplinary Appl. Math. 24, Springer-Verlag, 2003.

[3] Bloch, A. M., M. Leok, J. E. Marsden, and D. V. Zenkov [2005], Controlled Lagrangians and Stabilization of the Discrete Cart-Pendulum System, Proc. CDC 44, 6579-6584.

[4] Bloch, A. M., N. Leonard, \& J.E. Marsden, Stabilization of Mechanical Systems Using Controlled Lagrangians, Proc. CDC 36, 1997, 2356-2361.

[5] Bloch, A. M., N. Leonard, \& J. E. Marsden, Matching and Stabilization by the Method of Controlled Lagrangians, Proc. CDC 37, 1998, 14461451.

[6] Bloch, A. M., N. Leonard, \& J.E. Marsden, Potential Shaping and the Method of Controlled Lagrangians, Proc. CDC 38, 1999, 1652-1657.

[7] Bloch, A. M., N. E. Leonard, \& J.E. Marsden, Controlled Lagrangians and the Stabilization of Mechanical Systems I: The First Matching Theorem, IEEE Trans. on Systems and Control 45, 2000, 2253-2270.

[8] Bloch, A. M., D-E. Chang, N. E. Leonard, \& J. E. Marsden, Controlled Lagrangians and the Stabilization of Mechanical Systems II: Potential Shaping, Trans. IEEE on Autom. Contr. 46, 2001, 1556-1571.

[9] Chang, D-E., A. M. Bloch, N.E. Leonard, J.E. Marsden, \& C. Woolsey, The Equivalence of Controlled Lagrangian and Controlled Hamiltonian Systems, Control and the Calculus of Variations (special issue dedicated to J. L. Lions) 8, 2002, 393-422.

[10] Guibout, V. \& A.M. Bloch, A Discrete Maximum Principle for Solving Optimal Control Problems, Proc. CDC 43, 2004, 1806-1811.

[11] Hamberg, J., General Matching Conditions in the Theory of Controlled Lagrangians, Proc. CDC 38, 1999, 2519-2523.

[12] Hamberg, J., Controlled Lagrangians, Symmetries and Conditions for Strong Matching, In: Lagrangian and Hamiltonian Methods for Nonlinear Control, Elsevier, 2000.

[13] Junge, O., J. Marsden, \& S. Ober-Blbaum, Discrete Mechanics and Optimal Control, Proc. of the 16th IFAC World Congress, 2005.

[14] Kane, C., J.E. Marsden, M. Ortiz, \& M. West, Variational Integrators and the Newmark Algorithm for Conservative and Dissipative Mechanical Systems, Int. J. Numer. Math. Eng. 49, 2000, 1295-1325.

[15] Marsden, J.E., Lectures on Mechanics, London Mathematical Society Lecture Note Series 174, Cambridge University Press, 1992.

[16] Marsden, J.E. \& T.S. Ratiu, An Introduction to Mechanics and Symmetry. Texts in Appl. Math. 17, Springer-Verlag, 1999.

[17] Marsden, J.E. \& M. West, Discrete Mechanics and Variational Integrators, Acta Numerica 10, 2001, 357-514.

[18] Maschke, B., R. Ortega, \& A. van der Schaft, Energy-Based Lyapunov Functions for Forced Hamiltonian Systems with Dissipation, IEEE Trans. Automat. Control 45, 2001, 1498-1502.

[19] Sanyal, A., J. Shen, N. H. McClamroch, \& A. M. Bloch, Stability and Stabilization of Relative Equilibria of the Dumbbell Satellite in Central Gravity, 2006, Journal of the American Institute of Aeronautics and Astronautics, (to appear).

[20] Zenkov, D. V., A. M. Bloch, N. E. Leonard, \& J. E. Marsden, Matching and Stabilization of Low-Dimensional Nonholonomic Systems, Proc. CDC 39, 2000, 1289-1295.

[21] Zenkov, D. V., A. M. Bloch, \& J.E. Marsden, Flat Nonholonomic Matching, Proc. ACC, 2002, 2812-2817. 